\title{
A study on incidence and associated factors of retinopathy of prematurity in a tertiary care setting in Sri Lanka
}

\author{
*Indika Liyanage ${ }^{1}$, K S Y Perera ${ }^{2}$, Medha Weerasekera ${ }^{3}$
}

Sri Lanka Journal of Child Health, 2017; 46(4): 322-325

\begin{abstract}
Introduction: Retinopathy of prematurity (ROP) chiefly affects preterm infants. With the increased survival of extremely preterm infants, the number of infants with severe ROP has also increased.
\end{abstract}

Objective: To assess the incidence, associated factors and outcome of ROP in neonates born at less than 34 weeks of gestation and/or less than $1.5 \mathrm{~kg}$ birth weight in Sri Jayawardenepura General Hospital

Method: A prospective, descriptive, observational study was conducted in neonates born from $01 / 01 / 2014$ to $31 / 12 / 2014$, who were less than 34 weeks of gestation and/or less than $1.5 \mathrm{~kg}$ birth weight. Data was collected from patient records. Incidence estimates were given using descriptive statistics. For bivariate analysis (where associated factors were considered), the Chi Square test was applied to assess significant associations. The level of significance was 0.05 .

Results: Of 126 neonates, 113 were included in study as 11 met exclusion criteria and 2 dropped out during follow up. Twenty eight (24.8\%) neonates had ROP of whom $12(42.9 \%)$ needed treatment. Study revealed that, earlier the gestation and lower the birth weight, ROP was more commonly observed. Patent ductus arteriosus (PDA) $(\mathrm{p}=0.006)$, sepsis $(\mathrm{p}=0.024)$, invasive ventilation for 7 or more days $(\mathrm{p}=0.0001)$ and supplementary oxygen for more than 7 days $(\mathrm{p}=0.0001)$ were significantly associated with development of ROP.

${ }^{1}$ Senior Registrar in Neonatology, Castle Street Hospital for Women, Sri Lanka, ${ }^{2}$ Medical Officer, Sri Jayawardenepura General Hospital, Sri Lanka, ${ }^{3}$ Consultant Neonatologist, Sri Jayawardenepura General Hospital, Sri Lanka

*Correspondence: ikaliyanage@gmail.com (Received on 03 February 2017: Accepted after revision on 17 March 2017)

The authors declare that there are no conflicts of interest

Personal funding was used for the project.

Open Access Article published under the Creative Commons Attribution CC-BY (CC) (i)
Conclusions: Incidence of ROP at Sri Jayawardenapura General Hospital in 2014 was 248/1000 live births of neonates less than 34 weeks gestation and/or birth weight less than $1.5 \mathrm{~kg}$. PDA, sepsis, invasive ventilation for more than 7 days and oxygen supplementation for more than 7 days were significantly associated with occurrence of ROP.

DOI: http://dx.doi.org/10.4038/sljch.v46i4.8378

(Key words: Retinopathy of prematurity, ROP screening, preterm neonates)

\section{Introduction}

Retinopathy of prematurity (ROP) chiefly affects preterm infants ${ }^{1}$. With the increased survival of extremely preterm infants, the number of infants with severe ROP has also increased ${ }^{2,3}$. The international classification of ROP (ICROP) was first published in 1984 and later expanded in 1987. Further aspects of ICROP were revised and published in 2005. ICROP describes 5 stages $^{2,3}$ :

- Stage I is characterized by a line of demarcation. Extra vessels can be seen growing at the leading edge of the retinal vasculature. The line of demarcation separates the vascularized portion of the retina from the anteriorly positioned avascular retina.

- Stage II is characterized by an elevated ridge, rather than a flat demarcation line. Neovascularization may be present but is posterior to the ridge.

- Stage III refers to extra-retinal neovascularization or vessels that grow on to the ridge and then into the vitreous (extra retinal fibrovascular proliferation).

- $\quad$ Stage IV refers to partial retinal detachment.

- Stage $\mathrm{V}$ is total retinal detachment.

In addition, presence of aggressive posterior ROP, pre-plus disease and plus disease are included in the classification. In our study these sub-classifications were not analysed. 


\section{Objectives}

To study premature neonates born in a tertiary care centre in Sri Lanka to demonstrate the incidence of ROP and the risk factors of that population.

\section{Method}

A prospective, descriptive observational study was conducted in the neonatal intensive care unit (NICU) at Sri Jayawardenepura General Hospital. All neonates born from $01 / 01 / 2014$ to $31 / 12 / 2014$ who were less than 34 weeks of gestational age and/or less than $1.5 \mathrm{~kg}$ in birth weight were included in the study. Of them, neonates who expired during first 28 days of life (before $1^{\text {st }}$ ophthalmological assessment for ROP), neonates with other ophthalmological illness and neonates with severe congenital anomalies were excluded from the study.

Data was collected from patient clinical records and ophthalmological assessment records of ROP. The study was approved by the Ethical Review Committee of Sri Jayawardenepura General Hospital. Informed written consent was obtained from parents or guardian of each patient.

All neonates born in Sri Jayawardenepura General Hospital, who were less than 34 weeks of gestational age and/or less than $1.5 \mathrm{~kg}$ in birth weight were referred for detailed ophthalmological assessment by a Consultant Ophthalmologist, when they reached the $28^{\text {th }}$ day of life. First ophthalmological assessment for the neonates admitted to the NICU was done in the Ophthalmology Clinic or in NICU at Sri Jayawardenepura General Hospital, on Monday or Wednesday, whichever came first, when a neonate reached 28 days. Retinal ROP screening examinations were done after pupillary dilation by using binocular indirect ophthalmoscopy by the ophthalmologist. Findings of ROP examinations were recorded according to the ICROP. Presence of any stage of ROP was considered as having ROP for the purpose of this study. All details of the ophthalmological assessment were entered into a standard clinic record which was kept with the patient's clinical notes during the hospital stay and given to the parents on discharge, which includes information about the stage, zone and extent of any ROP and the presence of any pre-plus or plus disease. When further examination was required, the need for and time of this examination was also documented.

Incidence estimates were given using descriptive statistics. For bivariate analysis (where associated factors were considered), the Chi Square test was applied to assess significant associations. (Here the outcome was considered categorical like having ROP or not having it) The level of significance was 0.05 .

\section{Results}

Total number of neonates born from $01 / 01 / 2014$ to 31/12/2014 at Sri Jayawardenepura General Hospital, who were less than 34 weeks of gestation and/or less than $1.5 \mathrm{~kg}$ birth weight was 126 of whom 113 neonates were included in the study, as 11 met exclusion criteria and 2 dropped out during follow up. Twenty eight (24.8\%) neonates had ROP of whom $12(42.9 \%)$ needed treatment.

Figure 1 demonstrates the distribution of neonates with ROP according to gestational age. Figure 2 demonstrates the distribution of neonates with ROP according to their birth weight. ROP was more commonly observed in neonates who were born at an early gestation and/or with lower birth weight.

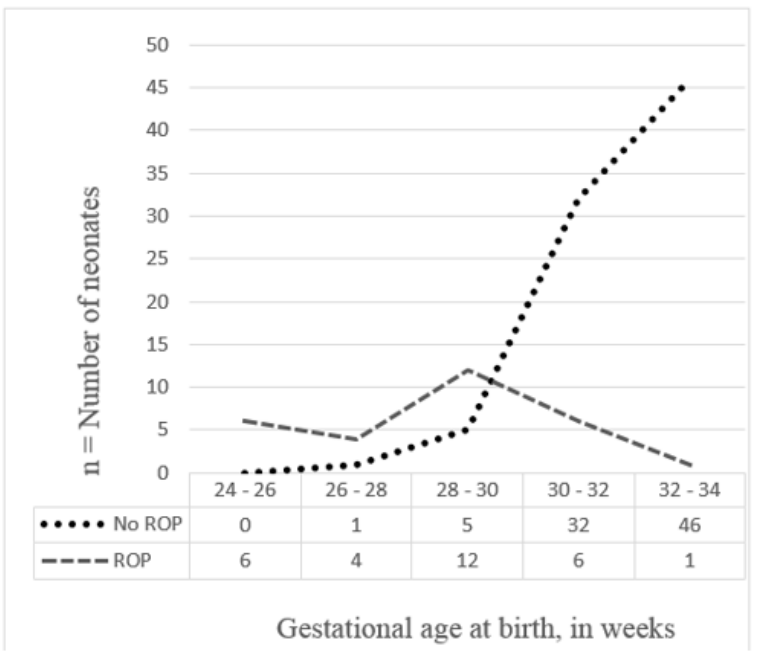

Figure 1: Distribution of ROP according to gestational age at birth

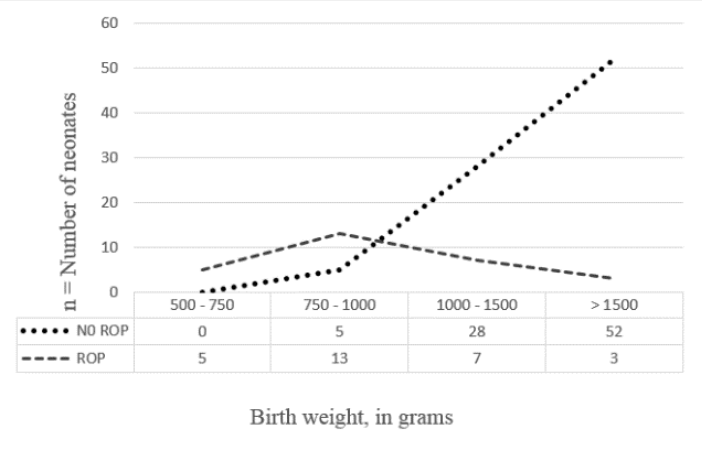

Figure 2: Distribution of ROP according to the birth weight

The proportion of PDA was higher among neonates with ROP $(n=5,17.8 \%)$ than with neonates without ROP $(\mathrm{n}=1,1.2 \%)$. The association between having a 
PDA and having ROP was statistically significant (Chi square 11.656, $\mathrm{p}=0.006$ ). The proportion of sepsis was higher among neonates with ROP $(n=4$, $14.3 \%)$ than with neonates without ROP $(n=6,7.1 \%)$. The association between having a diagnosis of sepsis and ROP was statistically significant $(\mathrm{p}=0.024)$.

The proportion who needed ventilator support for more than 7 days had higher occurrence of ROP $(\mathrm{n}=18,64.3 \%)$ whereas the proportion who needed no ventilator support or less than 7 days of ventilator support had lesser occurrence of ROP $(n=10,35.7 \%)$. There was a statistically significant $(\mathrm{p}=0.0001)$ difference between the two groups. The association between occurrence of ROP and need for ventilatory support was not significant when the cut-off used for ventilatory support was considered to be 3 days. Therefore prolonged invasive ventilation (more than 7 days) demonstrated a statistically significant association with occurrence of ROP among the study population.

The proportion who needed supplementary oxygen for more than 7 days had higher occurrence of ROP $(\mathrm{n}=25,89.3 \%)$ whereas the proportion who needed no supplementary oxygen or less than 7 days of supplementary oxygen had lesser occurrence of ROP $(n=3,10.7 \%)$. There was a statistically significant $(p=0.0001)$ difference between the two groups.

\section{Discussion}

In a study done on early presentation of severe ROP in Sri Lanka by Abeysekera H, et al in 2014 at the Eye Unit of the Lady Ridgeway Hospital for Children, $214(37.6 \%)$ babies had some degree of ROP ${ }^{4}$. From this group only $106(18.7 \%)$ had threshold ROP and required treatment. Sample size was 568, which included total number of babies screened at the Eye Unit of the Lady Ridgeway Hospital for Children during June 2013 and May 2014. A study done in the Teaching Hospital Peradeniya (THP) over a 16 month period from February 2003 to determine the incidence of ROP in the Neonatal Intensive Care Unit of THP revealed that 15 out of 106 screened neonates had significant ROP (i.e. stage 3 and beyond) 5 .

Mansouri M, et al. in 2014 showed that there was a significant difference regarding mechanical ventilation between the ROP and non ROP groups ${ }^{6}$. In a study done by Bayat-Mokhtari M, et al. in 20062007, out of 199 preterm neonates, $9.5 \%$ had ROP needing laser therapy, 33\% had ROP regressing spontaneously and $58 \%$ had no $\mathrm{ROP}^{7}$. Risk factors included gestational age, birth weight, Apgar score at one minute, mean duration of mechanical ventilation and mean duration of oxygen therapy ${ }^{7}$.
In a study conducted by Ortega-Molina et al., using data of three cohort studies in Spain, genetics, environment, and chance were responsible for $78.8 \%$, $23.8 \%$ and $4.1 \%$ of ROP cases, respectively ${ }^{8}$. Among the environmental factors, the number of intubation days, postpartum weight gain and sepsis were the highest risk factors for ROP, respectively ${ }^{8}$. In our study, PDA, sepsis, prolonged ventilation more than 7 days, prolonged oxygen therapy more than 7 days were found to have statistically significant association with ROP.

The American Academy of Paediatrics, the American Academy of Ophthalmology and the American Association of Paediatric Ophthalmology and Strabismus have established the screening instructions that are currently used in neonatal intensive care units (NICUs) ${ }^{9}$. According to these studies, all preterm infants born at less than 30 weeks of gestation and/or with a birth-weight less than $1500 \mathrm{~g}$ should undergo indirect ophthalmoscopy of dilated eyes ${ }^{9}$. The ophthalmoscopy should start from the 31st week of post conception age or from the 28th day of life ${ }^{9}$. According to UK guidelines all babies less than 32 weeks gestational age (up to 31 weeks and 6 days) or less than $1501 \mathrm{~g}$ birth weight should be screened for ROP ${ }^{10}$.

Since our study was done only in one hospital, it is difficult to predict about overall local figures using the analysed data. Therefore similar studies including several centres would be beneficial in the future. One limitation of our study was that we only assessed one of the short term outcomes of ROP, which was whether treatment was needed or not. In future studies, it would be beneficial if short term outcome was categorized into different levels of severity and the mode of treatment as well as the long term outcome of ROP were assessed to get a better understanding of the gravity of impact.

\section{Conclusions}

Incidence of ROP at Sri Jayawardenepura General Hospital in 2014 was 248/1000 live births of neonates less than 34 weeks gestation and/or birth weight less than $1.5 \mathrm{~kg}$. PDA, sepsis, invasive ventilation for more than 7 days and oxygen supplementation for more than 7 days were significantly associated with occurrence of ROP.

\section{Acknowledgements}

Our sincere gratitude to Dr. D. Wariyapola, Consultant Ophthalmologist, Sri Jayawardenapura General Hospital for the advice and guidance provided for this study. 


\section{References}

1. Alme AM, Mulhern ML, Hejkal TW, Meza JL, Qiu F, Ingvoldstad DD, et al. Outcome of retinopathy of prematurity patients following adoption of revised indications for treatment. BMC Ophthalmology 2008; 8(23): 1471-2415. https://doi.org/10.1186/1471-2415-8-23

2. International Committee for the classification of the late stages of retinopathy of prematurity: An international classification of retinopathy of prematurity. II. The classification of retinal detachment. Archives of Ophthalmology 1987; 105:906-12.

https://doi.org/10.1001/archopht.1987.01060070 042025

PMid: 3606449

3. International Classification of Retinopathy of Prematurity, Revisited. Archives of Ophthalmology 2005; 123 (7): 991-9.

https://doi.org/10.1001/archopht.1987.01060070 042025

PMid: 3606449

4. Abeysekera H, Irugalbandara D, Fonseka C. Early presentation of severe ROP in Sri Lanka. The Journal of the College of Ophthalmologists of Sri Lanka 2015; 21: 16-21.

5. Kiridana V, Wickremasingha N. An observational study on retinopathy of prematurity in the neonatal intensive care unit at Teaching Hospital, Peradeniya, Sri Lanka. Sri Lanka Journal of Child Health, 2010; 39:49-52. https://doi.org/10.4038/sljch.v39i2.1957

6. Mansouri M, Hemmatpour S, Sedighiani F, Ghamari M, Chavoshi D. Factors associated with retinopathy of prematurity in hospitalized preterm infants in Sanandaj, Iran. Electron Physician 2016; 8(9): 2931-4.

https://doi.org/10.19082/2931

PMid: 27790346 PMCid: PMC5074752
7. Bayat-Mokhtari M, Pishva N, Attarzadeh A, Hosseini H, Shahnaz $\mathrm{P}$. Incidence and risk factors of retinopathy of prematurity among preterm infants in Shiraz/Iran. Iranian Journal of Pediatrics 2010; 20(3): 303-7.

PMid: 23056721 PMCid: PMC3446051

8. Ortega-Molina JM, Anaya-Alaminos R, UberosFernández J, Solans-Pérez de Larraya A, Chaves Samaniego MJ, Salgado-Miranda A, et al. Genetic and environmental influences on retinopathy of prematurity. Mediators of Inflammation 2015; 2015: 764159

https://doi.org/10.1155/2015/764159

PMid: 26089603 PMCid: PMC4454750

9. Fierson W.M., American Academy of Pediatrics Section on Ophthalmology, American Academy of Ophthalmology, et al: Screening examination of premature infants for retinopathy of prematurity. Pediatrics 2013; 131(1): 189-195. https://doi.org/10.1542/peds.2012-2996 PMid: 23277315

10. Guideline for the Screening and Treatment of Retinopathy of Prematurity, May 2008. Royal College of Paediatrics and Child Health, Royal College of Ophthalmologists British Association of Perinatal Medicine \& BLISS. 